\title{
MULTIPLICACIÓN CLONAL DEL ÁRBOL DE LA QUINA (Cinchona officinalis L.): UNA ALTERNATIVA PARA CONSERVAR EL ÁRBOL NACIONAL DE PERÚ
}

\author{
Tito Sánchez-Santillan ${ }^{1}$, Jegnes B. Meléndez-Mori ${ }^{2}$, Eli Morales-Rojas ${ }^{2}$, \\ Ariel K. Chichipe-Puscan ${ }^{1}$, Segundo M. Oliva-Cruz ${ }^{2}$ y María H. Huaman-Vela ${ }^{3}$
}

\begin{abstract}
RESUMEN
El árbol de la quina (Cinchona officinalis), especie emblemática de Perú, durante muchos años ha venido siendo amenazado de desaparecer. El objetivo de este estudio fue evaluar la influencia del tipo de mini-estaca (apical y basal) y el tiempo de absorción de ácido indolbutírico (0, 2, 6 y 10 minutos) sobre la multiplicación clonal del árbol de la quina. Los brotes fueron colectados de árboles de poblaciones naturales presentes en el bosque montano Conila-Perú, a una altitud de $2800 \mathrm{~m}$. Las mini-estacas, basales y apicales, se trataron con ácido indolbutírico a $2000 \mathrm{mg} \cdot \mathrm{L}^{-1}$ y se dejaron inmersas por distintos tiempos. Los datos se sometieron a un análisis de varianza y las medias se compararon con la prueba Tukey. Luego de 60 días se encontró que las miniestacas basales presentaron un porcentaje de enraizamiento significativamente superior $(54,77 \%)$ respecto a las mini-estacas apicales $(40,10 \%)$, siendo superior también en sobrevivencia $(62,75 \%)$ y tamaño de raíces $(17,38 \mathrm{~mm})$. Los tiempos de 6 y 10 minutos de absorción fueron los más sobresalientes, mientras que el control ( 0 minutos) resultó ser el tratamiento más bajo. En conclusión, el uso de mini-estacas basales y tiempos de absorción de 6 y 10 minutos son las mejores condiciones para garantizar el enraizamiento de las mini-estacas del árbol de la quina.

Palabras clave adicionales: Ácido indolbutírico, brotes plagiotrópicos, enraizamiento, propagación vegetativa
\end{abstract}

\begin{abstract}
Clonal multiplication of the cinchona tree (Cinchona officinalis L.): an alternative to conserve the national tree of Peru The cinchona tree (Cinchona officinalis), an emblematic species of Peru, has for many years been threatened with extinction. The objective of this study was to evaluate the influence of the type of mini-stake (apical and basal) and the absorption time of indolebutyric acid $(0,2,6$, and 10 minutes) on the clonal multiplication of the cinchona tree. The shoots were collected from trees of natural populations present in the Conila-Peru montane forest, at an altitude of $2800 \mathrm{~m}$. The mini-cuttings, basal and apical, were treated with indole butyric acid at $2000 \mathrm{mg} \cdot \mathrm{L}^{-1}$ and were left to rest for different times. The data were subjected to an analysis of variance and the means were compared with the Tukey test. After 60 days, it was found that the basal mini-cuttings presented a significantly higher rooting percentage $(54.77 \%)$ compared to the apical mini-cuttings $(40.10 \%)$, being also higher in survival $(62.75 \%)$ and size of roots $(17.38 \mathrm{~mm})$. The absorption times of 6 and 10 minutes turned out to be the most outstanding, while the control ( 0 minutes) turned out to be the lowest treatment. In conclusion, the basal mini-cuttings and the absorption times of 6 and 10 minutes turn out to be the most appropriate to guarantee the rooting of mini-cuttings of the cinchona tree.
\end{abstract}

Additional keywords: Indole butyric acid, plagiotropic shoots, rooting, vegetative propagation

\section{INTRODUCCIÓN}

Cinchona officinalis, conocida como "árbol de quina" o "cascarilla", es una especie con importantes propiedades medicinales, perteneciente a la familia Rubiaceae (Raheem et al., 2004). En Perú, se considera un recurso vegetal emblemático de la flora del país debido a que simboliza la diversidad vegetal del territorio peruano. Además, posee gran valor económico debido al contenido de quinina,

Recibido: Enero 14, 2021

Aceptado: Julio 2, 2021

${ }^{1}$ Instituto de Investigaciones de la Amazonia Peruana, Iquitos-Loreto. e-mail: titosanchezsantillan@gmail.com (autor de correspondencia); achichipe@iiap.gob.pe

${ }^{2}$ Instituto de Investigación para el Desarrollo Sustentable de Ceja de Selva (INDES-CES), Universidad Nacional Toribio Rodríguez de Mendoza (UNTRM), Chachapoyas 01001, Perú. e-mail: jbenjamin@indes-ces.edu.pe; eli.morales@untrm.edu.pe; soliva@indes-ces.edu.pe

${ }^{3}$ Servicios Generales Jucusbamba EIRL. Luya, Perú. e-mail: marihuamanv170@gmail.com 
alcaloide ampliamente reconocido por su uso en la prevención y control de la malaria (Achan et al., 2011; Canales et al., 2020). La quina se distribuye a lo largo de zonas altoandinas y amazónicas de países como Bolivia, Ecuador, Colombia, Venezuela y Perú (Gómez et al., 2016; Barrutia et al., 2020). En Perú, el hábitat de la quina es el bosque nuboso, con clima cálido y húmedo, y precipitaciones abundantes y persistentes (Zevallos, 1989). Sin embargo, actualmente la necesidad del uso de tierras para la expansión agropecuaria junto con la deforestación vienen amenazando su hábitat, en consecuencia, el panorama para la conservación de especies del género Cinchona es incierto (Huamán et al., 2019; Vásquez et al., 2018).

La necesidad de recuperar y conservar esta especie emblemática plantea la importancia de reunir esfuerzos para su propagación. En condiciones naturales la supervivencia de la quina depende de la dispersión de sus semillas, pero desafortunadamente, aun cuando su tamaño facilita el proceso de diseminación, su capacidad para regenerar nuevas plántulas puede ser reducida o insuficiente, debido principalmente a que las semillas pierden viabilidad con rapidez (González et al., 2018; Lima et al., 2018). En ese sentido, la propagación sexual ha reportado resultados poco favorables para la germinación $(<50 \%)$ en condiciones de invernadero (Vásquez et al., 2018; Barrutia et al., 2020) pero además, las plántulas propagadas por esta vía pueden requerir de una permanencia prolongada en vivero para alcanzar el desarrollo óptimo para instalarlas en campo.

Para superar las limitaciones de su propagación se presenta como alternativa viable la propagación vegetativa a través de acodos, estacas y/o brotes (Conde et al., 2017), pues a pesar de los esfuerzos realizados para su cultivo in vitro (Moreno et al., 2018; Eras et al., 2019), el uso de herramientas biotecnológicas requiere mayor infraestructura, logística y recursos económicos. Los métodos de propagación convencional (como las estacas) permiten regenerar plántulas idénticas al progenitor a partir de un segmento de tallo que tiene la capacidad de formar raíces, brotes y hojas (Osuna et al., 2017). Sin embargo, la propagación de quina a través de estacas es considerado un proceso complejo, debido a que existen factores que influyen en la iniciación y emisión radicular, como por ejemplo la capacidad del material vegetal para absorber la fitohormona aplicada, sea por los tejidos nuevos (estacas apicales) o por la presencia de peridermis inicial (estacas subapicales) que poseen (Ramírez et al., 2004); así como también por el tiempo necesario para la absorción de la auxina (Vargas et al., 1999), pudiendo variar para cada especie.

Por lo anterior, la propagación de la quina, una especie emblemática para el país, requiere de estudios minuciosos para obtener plantones necesarios para su repoblamiento, sobre todo por las limitadas experiencias en el tema. Bajo ese contexto, el presente estudio tiene por objetivo evaluar la influencia del tipo de mini-estaca y el tiempo de absorción de auxina (ácido indolbutírico) sobre la multiplicación clonal del árbol de la quina utilizando microtúneles.

\section{MATERIALES Y MÉTODOS}

El experimento se realizó en las instalaciones de la empresa Servicios Generales Jucusbamba EIRL, ubicado en el distrito de Conila, región Amazonas (Perú). En el vivero se acondicionó un microtúnel con temperatura promedio de $17,5{ }^{\circ} \mathrm{C}$ y dimensiones de $6,0 \times 1,5 \times 0,9 \mathrm{~m}$ de largo, ancho y alto, respectivamente. Para mantener la turgencia de las estaquillas, dentro del microtúnel se instaló un sistema de riego con nebulizadores. La frecuencia de riego estuvo en función a las condiciones del clima. En periodos soleados el riego se realizó dos veces al día, y en periodos lluviosos una vez al día cada dos días. El sistema se activó por un máximo de un minuto.

Selección del material vegetal. En el bosque montano del distrito de Conila, a una altitud de $2800 \mathrm{~m}$, se identificaron árboles de quina (aproximadamente de $5 \mathrm{~m}$ ) libre de síntomas de fitopatógenos. En ramas plagiotrópicas (sin floración) se recolectaron brotes tiernos, y de ellas se separaron dos tipos de corte (miniestacas apicales y basales). El material recolectado se envolvió en papel húmedo y se colocó en cajas térmicas para su transporte al invernadero.

Instalación del experimento. Las ramas se desinfectaron mediante inmersión por tres minutos en solución fungicida de Curtine M8 (Cymoxanil + mancozeb), a una concentración de $1,0 \mathrm{~g} \cdot \mathrm{L}^{-1}$. Luego se separaron mini-estacas $(7 \mathrm{~cm})$ apicales y basales, dejando en ambas dos hojas con el $50 \%$ de su área. El extremo base $(<0,5 \mathrm{~cm})$ de la mini- 
estaca se introdujo en una solución de 2000 $\mathrm{mg} \cdot \mathrm{L}^{-1}$ de ácido indolbutírico (diluido en alcohol al $96 \%$ ), y posteriormente se dejaron a temperatura ambiente $\left(15-18{ }^{\circ} \mathrm{C}\right)$ para evaluar los tiempos de absorción 2, 6 y 10 minutos y, un testigo 0 minutos. Finalmente, las mini-estacas se colocaron individualmente en bolsas de aproximadamente $560 \mathrm{~cm}^{3}$ con sustrato compuesto por una mezcla volumétrica de arena y turba, en partes iguales (Sánchez et al., 2020).

Evaluaciones. A las ocho semanas, se registró el número de mini-estacas vivas y enraizadas y se expresó como porcentaje de sobrevivencia y porcentaje de enraizamiento (calculado con base en el número inicial de estaquillas instaladas). Además, en cada mini-estaca, se cuantificó el número de raíces (solo aquellas raíces con una longitud igual o superior a $5 \mathrm{~mm}$ ) y con un vernier digital se midió la longitud de las raíces (promedio de todas raíces con una longitud igual o superior a $5 \mathrm{~mm}$ ) (Vásquez et al., 2018, Sánchez et al., 2020). La mini-estaca se consideró viva y enraizada cuando presentó al menos una raíz con una longitud mínima de $5 \mathrm{~mm}$.

Diseño experimental y análisis de datos. El experimento se instaló bajo un diseño completamente al azar con arreglo factorial $2 \times 4$, representado por 2 tipos de mini-estaca (apical y basal) y 4 tiempos de absorción (0, 2, 6 y 10 minutos). Se conformaron ocho tratamientos con tres repeticiones y 18 mini-estacas por unidad experimental.

Se verificó el cumplimiento de los supuestos de normalidad (Shapiro-Wilk) y homogeneidad de varianzas (Levene). Luego se realizó el análisis de varianza (ANOVA) y las medias fueron comparadas mediante la prueba de Tukey. Los datos se analizaron utilizando el programa estadístico InfoStat, versión 2017.

\section{RESULTADOS}

El análisis de varianza mostró que los factores tuvieron influencia significativa sobre el enraizamiento de mini-estacas de quina (Cuadro 1). La comparación múltiple por factores indicó que las mini-estacas basales alcanzaron promedios superiores $(P \leq 0,05)$ en cuanto al porcentaje de sobrevivencia, enraizamiento, número de raíces y longitud de raíces; por el contrario, las miniestacas apicales presentaron los valores más bajos para las variables antes señaladas (Cuadro 2).

Cuadro 1. Valores de probabilidad $(P)$ en el análisis de varianza de las variables de enraizamiento de mini-estacas de $C$. officinalis

\begin{tabular}{lcccc}
\hline \multicolumn{1}{c}{ Fuente de variación } & Sobrevivencia & Enraizamiento & Número de raíces & Longitud de raíces \\
\hline Tipo de mini-estaca & $0,0318^{*}$ & $0,0039^{* *}$ & $0,0002 * * *$ & $0,0000^{* * *}$ \\
$\begin{array}{l}\text { Tiempo de absorción } \\
\text { Tipo de mini-estaca x tiempo de }\end{array}$ & $0,7482 \mathrm{~ns}$ & $0,0216^{*}$ & $0,0006 * * *$ & $0,0001 * * *$ \\
absorción & $0,9634 \mathrm{~ns}$ & $0,8253 \mathrm{~ns}$ & $0,0004 * * *$ & $0,0299 *$ \\
\hline
\end{tabular}

$*=\mathrm{P} \leq 0,05 ; * *=P \leq 0,01 ; * * *=P \leq 0,001 ; \mathrm{ns}=$ no significativo

Cuadro 2. Efecto del tipo de estaca y el tiempo de absorción de ácido indolbutírico sobre la capacidad de enraizamiento de mini-estacas de C. officinalis

\begin{tabular}{lcccc}
\hline \multicolumn{1}{c}{ Factor } & $\begin{array}{c}\text { Sobrevivencia } \\
(\%)\end{array}$ & $\begin{array}{c}\text { Enraizamiento } \\
(\%)\end{array}$ & $\begin{array}{c}\text { Número } \\
\text { de raíces* }\end{array}$ & $\begin{array}{c}\text { Longitud de } \\
\text { raíces (mm)* }\end{array}$ \\
\hline Tipo de mini-estaca & & & & \\
Basal & $62,75 \mathrm{a}$ & $54,77 \mathrm{a}$ & $8,33 \mathrm{a}$ & $17,38 \mathrm{a}$ \\
Apical & $47,52 \mathrm{~b}$ & $40,10 \mathrm{~b}$ & $2,81 \mathrm{~b}$ & $13,29 \mathrm{~b}$ \\
Tiempo de absorción (minutos) & & & & \\
0 & $52,50 \mathrm{a}$ & $40,77 \mathrm{~b}$ & $1,78 \mathrm{c}$ & $12,98 \mathrm{~b}$ \\
2 & $52,57 \mathrm{a}$ & $43,77 \mathrm{ab}$ & $3,72 \mathrm{bc}$ & $14,32 \mathrm{~b}$ \\
6 & $54,23 \mathrm{a}$ & $44,50 \mathrm{ab}$ & $9,97 \mathrm{a}$ & $16,75 \mathrm{a}$ \\
10 & $61,23 \mathrm{a}$ & $60,70 \mathrm{a}$ & $6,82 \mathrm{ab}$ & $17,30 \mathrm{a}$ \\
\hline
\end{tabular}

Letras distintas en columnas dentro de cada factor indican diferencias significativas con la prueba Tukey $(P \leq 0,05)$.

*Evaluación del efecto simple 
Estos resultados muestran que las mini-estacas basales presentaron mejor comportamiento sobre el enraizamiento de quina, en comparación con las mini-estacas apicales, lo cual es particularmente notorio al cotejar el número de raíces (Figura 1). Por su parte, la comparación de los tiempos de absorción de la auxina muestra que el porcentaje de enraizamiento y el desarrollo radicular fue favorecido por los periodos de 6 y 10 minutos, es decir, conforme bajó el tiempo de absorción se redujo la emisión y crecimiento de raíces (Cuadro 2).
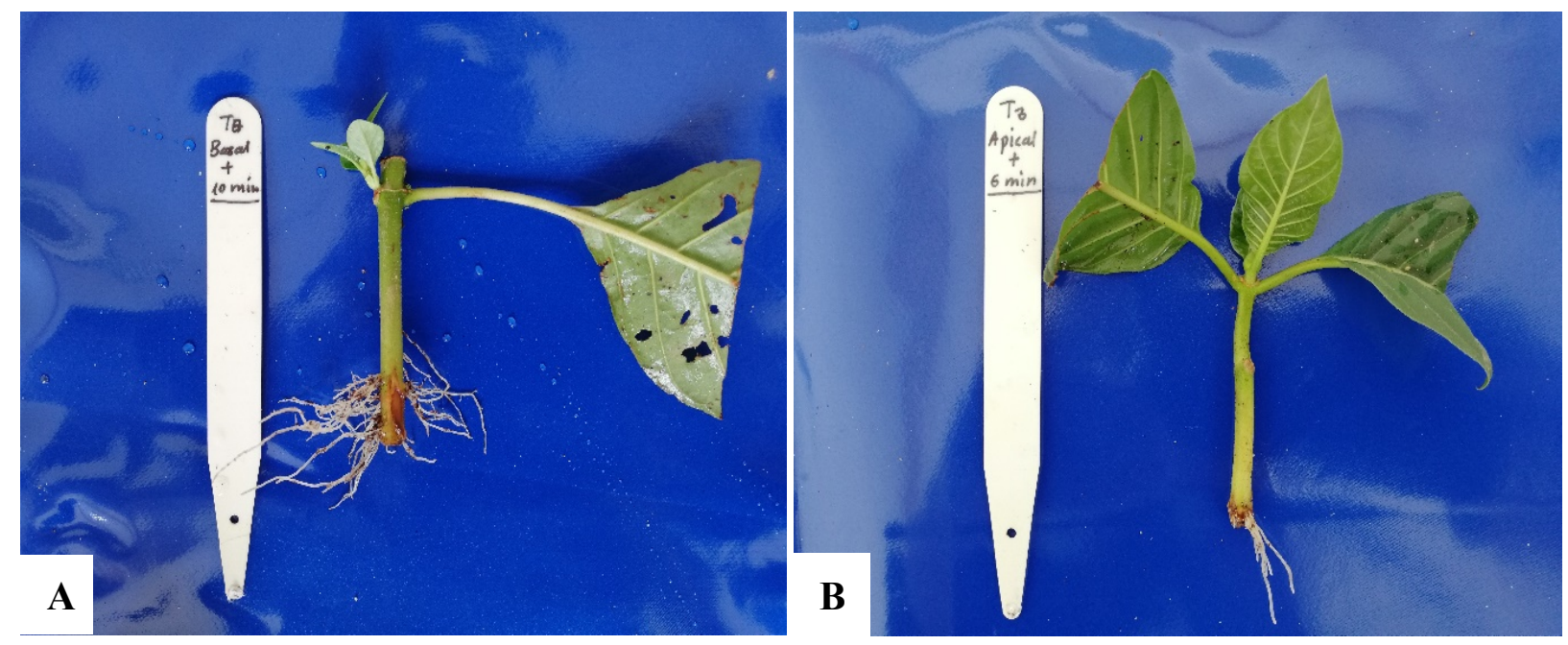

Figura 1. Muestra del enraizamiento de mini-estacas de C. officinalis a los 60 días. A) mini-estaca basal y B) mini-estaca apical

En la evaluación de la interaccion de factores hubo diferencias significativas en cuanto al número y longitud de raíces, mientras que para la sobrevivencia y enraizamiento de miniestacas no hubo diferencia (Cuadro 1). El mayor número de raíces, se alcanzó en la interacción entre mini-estacas basales y un tiempo de absorción de 6 minutos (Figura 2A), registrando un promedio de 17,47 raíces. Por el contrario, los tratamientos con mini-estacas aplicales presentaron los promedios más bajos, no superando la formación de cuatro raíces por miniestaca. En cuanto a la longitud de raíz, se evidenció que la interacción entre mini-estacas basales y un tiempo de absorción de 10 minutos favoreció al crecimiento de raíces, alcanzando un promedio de 20,8 $\mathrm{mm}$ (Figura 2B).

\section{DISCUSIÓN}

La propagación vegetativa de $C$. officinalis muestra importantes diferencias en la capacidad de enraizamiento, según el tipo de estaca utilizada. Entre las variables evaluadas se evidenció que la respuesta de las mini-estacas basales fue significativamente superior a los valores registrados en las apicales. Sin embargo, el porcentaje de enraizamiento obtenido tanto en las mini-estacas apicales $(40,10 \%)$ como en las miniestacas basales $(54,77 \%)$ se puede considerar como una capacidad de enraizamiento media, ya que según la escala propuesta por Del Río y Caballero (2005) en esta categoría, la tasa de enraizamiento se encuentra entre 40 y $60 \%$. En quina, distintos estudios han evaluado el efecto de factores como el sustrato y las hormonas en el enraizamiento de esquejes, pero los resultados no han sido completamente favorables (Sánchez et al., 2020), y en investigaciones futuras, se podrían evaluar variables anatómicas, así como niveles de carbohidratos y compuestos fenólicos. Según Villar et al. (2018), las especies del género Cinchona presentan dificultad para enraizar y en ocasiones no logran formar callos, siendo una respuesta desfavorable que señalan como un probable efecto inhibitorio de los alcaloides que presenta el material vegetal y que pueden afectar todo tipo de proceso rizogénico.

En el caso del tipo de mini-estacas, existen controversias en la elección de una sección óptima 
de la rama para establecer ensayos de enraizamiento. Al respecto, se menciona que las estacas basales resisten mejor las condiciones adversas debido a su mayor lignificación, mientras que las apicales son propensas a sufrir alta mortalidad cuando no tiene condiciones favorables (Mesén, 1998). Además, las estacas

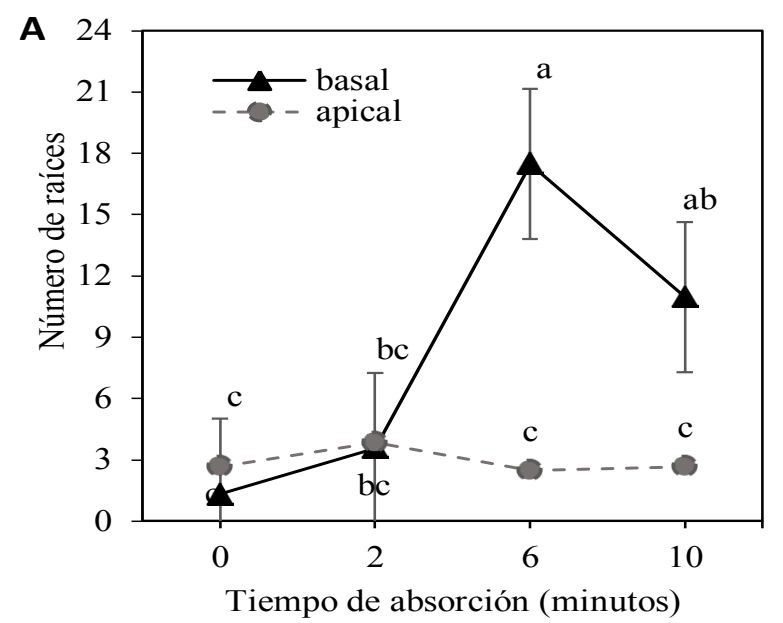

apicales contienen mayor porcentaje de agua en su estructura, por lo cual es probable que la fitohormona aplicada sea lavada por la exudación a través del corte realizado (Hartmann et al., 2014). Estos factores podrían explicar las diferencias reportadas en este estudio para ambos tipos de estacas.

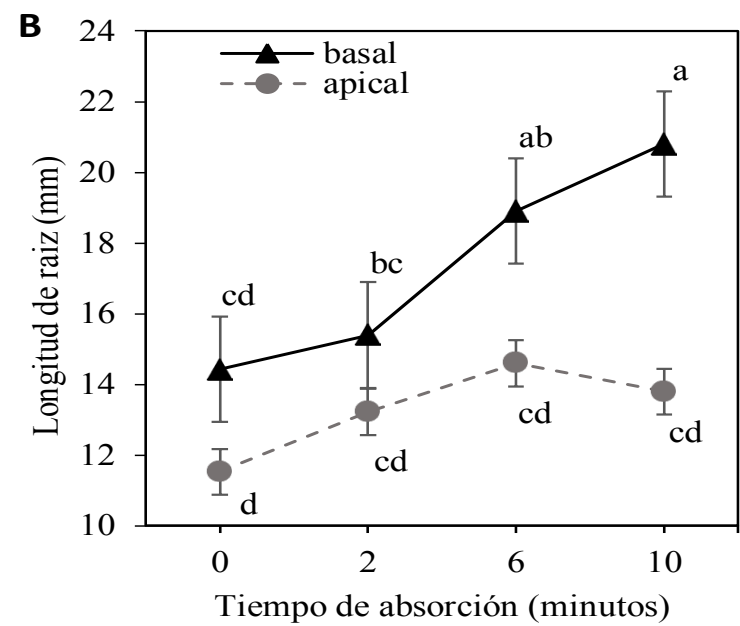

Figura 2. Efecto de la interacción entre el tipo de mini-estaca y el tiempo de absorción: A) sobre el número de raíces y B) sobre la longitud de raíces. Prueba Tukey $(P \leq 0,05)$. En cada punto se presentan las barras de error

Diferentes investigaciones han mostrado que el tipo de estaca tiene una influencia alta en el proceso de enraizamiento y puede variar según la especie que se desee propagar. Por ejemplo, en especies como Populus spp. (Zalesny et al., 2003) y Pinus leiophylla (Cuevas et al., 2015), las estacas basales mostraron un mayor nivel de enraizamiento en comparación con las estacas apicales e intermedias; por el contrario, en Ixora coccinea L. var. coccinea (Fernández et al., 2011) y Cedrela odorata (Sampayo et al., 2016), la tasa de enraizamiento y sobrevivencia fueron más altas en las estacas apicales. Por otro lado, en Gardenia jasminoides, el porcentaje de enraizamiento tanto para estacas apicales como basales registraron valores similares (Navarro et al., 2010). Estas variaciones pueden estar relacionadas por un lado con el grado de lignificación de las estacas basales que puede favorecer la absorción de auxinas debido a la menor emisión de exudados $\mathrm{y}$, por otra parte, se debe considerar que las estacas apicales concentran mayor cantidad de auxinas naturales y estimulan el desarrollo radical (Moore, 1984; Ruiz et al., 2005; Hartmann et al., 2014). En términos generales se puede mencionar que el nivel de juvenilidad, la lignificación y auxinas naturales son factores que influyen significativamente en el enraizamiento de estacas.

Por su parte, el tiempo de absorción muestra ser un factor importante en la capacidad de enraizamiento, pues los resultados indican que con el aumento del tiempo de absorción de ácido indolbutírico (al menos hasta períodos de 10 minutos) se incrementa tanto el porcentaje de enraizamiento y sobrevivencia como el número y longitud de raíces. En efecto, los tiempos de reposo o absorción de la hormona favorecieron la capacidad rizogénica de mini-estacas de $C$. officinalis. Este factor es poco estudiado en la propagación vegetativa, pero es sabido que las auxinas se pueden utilizar tanto en sólidos como en líquidos; siendo una característica que en ocasiones podrían ser una limitante debido a que estarían propensos al lavado por exudaciones del material vegetativo o por exceso del agua retenida en el sustrato (Hartmann et al., 2014).

El etanol utilizado para aplicar el ácido indolbutírico puede ser una sustancia que afecte 
negativamente la capacidad rizogénica de las mini-estacas, sobre todo en aquellas con tejido muy suculento. Según mencionan Cerveny y Gibson (2005), auxinas disueltas en este compuesto (alcohol) son más propensas a causar quemaduras y/o facilitar la deshidratación de los tejidos vegetales. Por lo tanto, es recomendable dejar evaporar el alcohol adherido al área de aplicación, lo cual permitirá una mejor absorción de la auxina (Sánchez et al., 2020). Toda vez que la absorción de auxinas en las mini-estacas es fundamental porque ayuda en el transporte de sustancias de reserva hacia la base de las miniestacas, favoreciendo el desarrollo radical (Mesén et al., 1997).

\section{CONCLUSIONES}

Este estudio evidencia que las mini-estacas basales presentan mayor sobrevivencia y desarrollo rizogénico respecto a las secciones apicales, siendo una alternativa viable para la propagación vegetativa de la quina utilizando microtuneles. Asimismo, se evidenció que los tiempos de absorción influyen en la capacidad de enraizamiento, presentando una relación directamente proporcional con la formación del sistema radicular de las mini-estacas.

\section{AGRADECIMIENTO}

Los autores agradecen a la empresa Servicios Generales Jucusbamba EIRL e Instituto de Investigaciones de la Amazonía Peruana, por el apoyo logístico para la ejecución del proyecto.

\section{LITERATURA CITADA}

1. Achan, J., A.O. Talisuna, A. Erhart, A. Yeka, J.K. Tibenderana, F.N. Baliraine, P.J. Rosenthal y U. D'Alessandro. 2011. Quinine, an old anti-malarial drug in a modern world: Role in the treatment of malaria. Malaria Journal 10: 144.

2. Barrutia-Rodríguez, R.R., I. Barrutia-Barreto y T.D. Marín-Velásquez. 2020. Germinación de semillas de Cinchona officinalis L. en tres tipos de suelos de Cajamarca, Perú. Revista Cubana de Ciencias Forestales 8(1): 75-87.

3. Canales, N.A., T.N. Gress-Hansen, C. Cornett,
K. Walker, F. Driver, A. Antonelli, C. Maldonado, M. Nesbitt, C.J. Barnes y N. Ronsted. 2020. Historical chemical annotations of Cinchona bark collections are comparable to results from current day high-pressure liquid chromatography technologies. Journal of Ethnopharmacology 249: 112375.

4. Cerveny, C. y J. Gibson. 2005. Grower 101: Rooting Hormones. Greenhouse Product News. https://n9.cl/uvbj4 (consulta de Sept. 10, 2020).

5. Conde, M.E., J.A. Moreno, V.H. Eras, J. Minchala, D. González, M. Yaguana y C. Valarezo. 2017. Multiplicación sexual y asexual de Cinchona officinalis L., con fines de conservación de la especie. Tzhoecoen 9(1): 81-93.

6. Cuevas-Cruz, J.C., M. Jiménez-Casas, J. JassoMata, P. Pérez-Rodríguez, J. López-Uptón y Á. Villegas-Monter. 2015. Asexual propagation of Pinus leiophylla Schiede ex Schltdl. et Cham. Revista Chapingo Serie Ciencias Forestales y del Ambiente 21(1): 81-95.

7. Del Río, C. y J.M. Caballero. 2005. Variabilidad y Selección: Aptitud al enraizamiento. In: Rallo, L., D. Barranco, J.M. Caballero, C. Del Río, A. Martín, J. Tous, I. Trujillo (eds.). Variedades de Olivo en España. Madrid, España. Mundiprensa. pp. 277-308.

8. Eras-Guamán, V., J. Moreno, M. Yaguana, R. Poma y D. Paredes. 2019. Balance hormonal para la fase de brotación y enraizamiento in vitro de explantes de Cinchona officinalis L., provenientes de relictos boscosos de la provincia de Loja. Bosques Latitud Cero 9(1): 58-68.

9. Fernández, J.L., F.M. Sosa, L. Castellanos, E. Casanovas y O. Alomá. 2011. Alternativas para el enraizamiento de estacas de Ixora coccinea L. var. coccinea. Centro Agrícola 38(2): 45-50.

10.Gómez, A., L.A. Beraun, O.J. Gómez y E. Llatas. 2016. Procesos de regeneración natural de la quina o cascarilla (Cinchona spp.): En los bosques de neblina del distrito de Kañaris, región Lambayeque. INIA. Estación Experimental Agraria Vista FloridaLambayeque. https://n9.cl/08ki (consulta de Sept. 10, 2020). 
11.González, K., V.H. Eras, J. Moreno, J. Minchala, M.Y. Yaguana y C.V. Valarezo. 2018. Procesos biotecnológicos para la inducción de callos a partir de vitroplantas de Cinchona officinalis L., a nivel de laboratorio en la provincia de Loja, Ecuador. Tzhoecoen 10(2): 299-312.

12.Hartmann, H.T., D.E. Kester, F.T. Davies y R.L. Geneve. 2014. Hartmann \& Kester's Plant Propagation: Principles and Practices. Eigth Edition. Pearson Education. Edinburgh Gate, Harlow, United Kingdom.

13.Huamán, L., J. Albán y E. Chilquillo. 2019. Aspectos taxonómicos y avances en el conocimiento del estado actual del árbol de la Quina (Cinchona officinalis L.) en el Norte de Perú. Ecología Aplicada 18(2): 145-153.

14.Lima, N.R., J.A. Moreno, V.H. Eras, J. Minchala, D. González, M. Yaguana y C. Valarezo. 2018. Propagación in vitro de Cinchona officinalis L. a partir de semillas. Revista de Investigaciones Altoandinas 20(2): 169-178.

15.Mesén, F, A.C. Newton y R.R. Leakey. 1997. Vegetative propagation of Cordia alliodora (Ruiz \& Pavon) Oken: The effects of IBA concentration, propagation medium and cutting origin. Forest Ecology and Management 92(1): 45-54.

16.Mesén, F. 1998. Enraizamiento de estacas juveniles de especies forestales: Uso de propagadores de sub-irrigación. Manual Técnico $\mathrm{N}^{\circ}$ 30. CATIE, Turrialba, Costa Rica $36 \mathrm{p}$.

17.Moore, G.M. 1984. Mechanisms of hormone action in plants. International Plant Propagators' Society 34: 79-90.

18.Moreno-Serrano, J.A., C. Pérez-Ruiz, I. Moreno-Fierro y J. Moreno-Fierro. 2018. Effect of culture medium on morphogenic processes in vitro in Cinchona officinalis L. Revista de la Facultad de Ciencias Agrarias UNCuyo 51(1): 55-68.

19. Navarro Sarría, B., F.M. Sosa, L. Castellanos, R. Soto, E. Casanovas y R. Hernández. 2010. Efecto del ácido indolacético sobre el enraizamiento de las estacas de Gardenia jasminoides W.N. Ellis. Centro Agrícola 37(4): 25-30.
20.Osuna, H.R., A.M. Osuna y A. Fierro. 2017. Manual de propagación de plantas superiores. México DF, México. Universidad Autónoma Metropolitana. $91 \mathrm{p}$.

21.Raheem, I.T., S.N. Goodman y E.N. Jacobsen. 2004. Catalytic asymmetric total syntheses of quinine and quinidine. Journal of the American Chemical Society 126(3): 706-707.

22.Ramírez-Villalobos, M., A. UrdanetaFernández y G. Vargas-Simón. 2004. Tratamientos con ácido indolbutírico y lesionado sobre el enraizamiento de estacas de icaco (Chrysobalanus icaco L.). Agronomía Tropical 54(2): 203-218.

23.Ruiz, R., J.J. Vargas, V.M. Cetina y Á.V. Monter. 2005. Efecto del ácido indolbutírico (AIB) y tipo de estaca en el enraizado de Gmelina arborea Roxb. Revista Fitotecnia Mexicana 28(4): 319-326.

24.Sampayo-Maldonado, S., M. Jiménez-Casas, J. López-Upton, V. Sánchez-Monsalvo, J. JassoMata, A. Equihua-Martínez y C.R. CastilloMartínez. 2016. Enraizado de miniestacas de Cedrela odorata L. Agrociencia 50(7): 919929.

25.Sánchez, T., G. Silva, A.K. Chichipe, M. Trigoso, L.G. Bobadilla y G.Y. Jiménez. 2020. Influencia de sustratos y concentraciones de ácido indolbutírico en la propagación vegetativa de Cinchona officinalis L. (quina) en Amazonas, Perú. Revista Cubana de Ciencias Forestales 8(2): 241-251.

26. Vargas-Simón, G., G. Arellano-Ostoa y R. Soto-Hernández. 1999. Enraizamiento de estacas de icaco (Chrysobalanus icaco L.) sometidas a aplicaciones de auxinas. Bioagro 11(3): 103-108.

27.Vásquez-Castañeda, J.H., E. Lápiz, M.K.Y. Barboza, S.N. Vásquez y L.M. Quispe. 2018. Comparación de sustratos en la propagación sexual y asexual del árbol de la quina (Cinchona officinalis). Revista de Investigación en Agroproducción Sustentable 2(3): 77-85.

28.Villar, M.Á., F.E. Marcelo y J.R. Baselly. 2018. Respuesta de la Cinchona officinalis L. al método de propagación asexual mediante estacas y esquejes. Instituto Nacional de Innovación Agraria. https://n9.cl/qiegg 
(consulta de Sep. 10, 2020.

29.Zalesny Jr., R.S., R.B. Hall, O.E. Bauer y D.E. Riemenschneider. 2003. Shoot position affects root initiation and growth of dormant unrooted cuttings of Populus. Silvae Genetica
52: 273-279.

30.Zevallos, P.A. 1989. Taxonomía, distribución geográfica y status del género Cinchona en el Perú. Lima, Perú. Universidad Nacional Agraria La Molina. 74 p. 